\title{
LETTERS
}

\section{Low-dose methotrexate in PAH related to T-cell large granular lymphocyte leukaemia}

\section{To the Editors:}

The classification of pulmonary hypertension $(\mathrm{PH})$ has recently been updated and $\mathrm{PH}$ associated with haematological disorders is classified as category $\mathrm{V}$ [1].

T-cell large granular lymphocytes (LGLs) are a morphologically distinct subset of normal peripheral blood mononuclear cells classified into two major lineages based on the presence of the CD3 receptor: $\mathrm{CD} 3+$ lymphocytes and CD3- natural killer cells. The revised European-American classification of lymphoid neoplasms [2] considers clonal proliferation of these cells, or LGL leukaemia (as proved by a cytogenetic study of the rearrangement of the Tcell receptor), as a distinct type of peripheral T-cell neoplasm. In the vast majority of cases, LGL leukaemia has a CD3+, $\alpha \beta+, \mathrm{CD} 4-$, CD8+, CD16+, CD57+ and CD56- phenotype and an indolent course [3]. Overexpression of some natural killer receptors (NKRs) of the killer immunoglobulin-like receptor family, such as CD94 and CD158b, is sometimes observed [4-6]. T-cell LGL leukaemia is frequently associated with various autoimmune diseases, particularly rheumatoid arthritis (30\% of cases) and haematological disorders (e.g. neutropenia) [3].

PH associated with T-cell LGL leukaemia has been described in rare cases and an aetiopathological link between the diseases has been suggested [4-8]. In this context, the efficacy of immunosuppressive agents in association with standard treatment (oral anticoagulants \pm diuretics \pm oxygen) with or without vasodilatory drugs has been investigated $[4,6,7]$.

Herein, we describe a new case of PH associated with T-cell LGL leukaemia that responded to immunosuppressive therapy. Possible aetiopathological mechanisms are discussed on the basis of literature data.

A 50-yr-old female without significant medical history was admitted to hospital because of rapidly progressive New York Heart Association (NYHA) functional class IV dyspnoea, with hypoxaemia (partial pressure of oxygen $60 \mathrm{mmHg}$ in ambient air) and right heart failure. Transthoracic echocardiography (TTE) revealed a severe elevation of pulmonary artery systolic pressure $(P$ pa,sys $)$ of $90 \mathrm{mmHg}$ without significant left ventricular dysfunction (ejection fraction $60 \%$ ) or primitive valvulopathy. The right heart cavities were dilated (ventricular diameter $>60 \mathrm{~mm}$ and ectasic auricle). Pre-capillary PH was confirmed by right heart catheterisation (RHC). The systolic, diastolic and mean pulmonary arterial pressures $\left(\bar{P}_{\mathrm{pa}}\right)$ were 90, 45 and $60 \mathrm{mmHg}$, respectively, mean pulmonary artery wedge pressure was $13 \mathrm{mmHg}$, the cardiac index $(\mathrm{CI})$ was $2 \mathrm{~L} \cdot \mathrm{min}^{-1} \cdot \mathrm{m}^{-2}$ and pulmonary vascular resistance (PVR) was 15.7 Wood units. An inhaled nitric oxide vasoreactivity test was negative. The 6-min walk distance (6MWD) was $80 \mathrm{~m}$. A clinical examination and biological and imaging investigations excluded other forms of pre-capillary $\mathrm{PH}$.

A blood cell count revealed haemolytic anaemia of uncertain origin (haemoglobin $10.1 \mathrm{~g} \cdot \mathrm{dL}^{-1}$, haptoglobin $0.08 \mathrm{mg} \cdot \mathrm{L}^{-1}$ and negative Coombs test) and leukoneutropenia (white blood cell count $3.2 \times 10^{9}$ cells $\cdot \mathrm{L}^{-1}$, polymorphonuclear cell count $0.8 \times 10^{9}$ cells $\left.\cdot \mathrm{L}^{-1}\right)$. Lymphocyte and platelet counts were normal. The patient received continuous nasal oxygen, an oral anticoagulant and intravenous dobutamine. 1 week later, dobutamine was stopped and continuous i.v. epoprostenol was initiated at a dose of $3 \mathrm{ng} \cdot \mathrm{kg}^{-1} \cdot \mathrm{min}^{-1}$ and gradually increased in increments of $1 \mathrm{ng}$. $\mathrm{kg} \cdot \mathrm{min}^{-1}$ to achieve a dose of $19 \mathrm{ng} \cdot \mathrm{kg}^{-1} \cdot \mathrm{min}^{-1}$ within $\sim 6$ weeks.

6 months later, the patient's clinical status had moderately improved on i.v. epoprostenol at the same dose and continuous oxygen therapy. The patient remained in NYHA functional class IV and was oxygen dependent. Her 6MWD was $251 \mathrm{~m}$. Ppa,sys measured by TTE was slightly reduced to $75 \mathrm{mmHg}$. A clinical examination revealed spleen enlargement. Worsening of neutropenia (white blood cell count $0.6 \times 10^{9}$ cells $\cdot \mathrm{L}^{-1}$ ), persistence of haemolytic anaemia (haemoglobin $10 \mathrm{~g} \cdot \mathrm{dL}^{-1}$ ) and mild T-cell LGL expansion of $0.3 \times 10^{9}$ cells $\cdot \mathrm{L}^{-1}$ on cytological analysis of a blood smear (15\% of lymphocyte count) were observed. Cytological analysis of a bone-marrow aspirate revealed LGL infiltration of $30 \%$. The immunophenotype of circulating lymphocytes, as assessed using a flow cytometric method, was CD3+, $\alpha \beta+, C D 4-, C D 8+, C D 16+, C D 57+, C D 158 b+, C D 94+$ and CD95+, which confirmed T-cell LGL expansion. A T-cell receptor rearrangement study by PCR established the clonality of the Tcell LGL proliferation. On the basis of this finding, oral low-dose methotrexate (15 mg per week) was initiated. 6 months after initiation of immunosuppressive treatment, the patient was in NYHA functional class I and her exercise capacity improved dramatically, as highlighted by an increase of her 6MWD to $550 \mathrm{~m}$. Ppa,sys measured by TTE decreased to $60 \mathrm{mmHg}$ and RHC revealed a significant improvement in all haemodynamic parameters: Ppa,sys $65 \mathrm{mmHg}, \bar{P}_{\text {pa }} 45 \mathrm{mmHg}, \mathrm{CI} 2.8 \mathrm{~L} \cdot \mathrm{min}^{-1} \cdot \mathrm{m}^{-2}$ and PVR 8.7 Wood units. Outpatient oxygen therapy was stopped. A complete haematological response was obtained, as shown by an increase in neutrophil count to $1.5 \times 10^{9}$ cells $\cdot \mathrm{L}^{-1}$ and a circulating LGL count that was $<10 \%$, with a normal circulating lymphocyte immunophenotype and the absence of T-cell LGL clone on cytogenetic analysis.

After the first 6 months of methotrexate treatment, the patient's symptoms dramatically improved and the epoprostenol dose was decreased to $15 \mathrm{ng} \cdot \mathrm{kg}^{-1} \cdot \mathrm{min}^{-1}$, but $P$ pa,sys measured by TTE increased to $90 \mathrm{mmHg}$ within 3 months. The previous epoprostenol dose of $19 \mathrm{ng} \cdot \mathrm{kg}^{-1} \cdot \mathrm{min}^{-1}$ was resumed and has not been decreased since. 
After 10 yrs of follow-up, the patient is taking i.v. prostacyclin and p.o. methotrexate at the same dose. Under this treatment she remains in NYHA functional class I, her 6MWD is $586 \mathrm{~m}$ and her Ppa,sys is stable at $55 \mathrm{mmHg}$.

T-cell LGL leukaemia is frequently associated with autoimmune diseases (notably seropositive rheumatoid arthritis) and haematological disorders (e.g. neutropenia, peripheral or central anaemia and thrombopenia associated with splenomegaly). In general, symptomatic T-cell LGL leukaemia responds to immunosuppressive treatment such as cyclosporine A, cyclophosphamide and methotrexate [3]. The course of T-cell LGL leukaemia is usually indolent, but aggressive disease has been reported and clinical observations of T-cell LGL leukaemia with severe $\mathrm{PH}$ that improved under immunosuppressive treatment have been reported $[4,6,7]$.

After 3 months of epoprostenol the clinical response was not satisfactory, as the patient remained in NYHA functional class IV with a low 6MWD $(251 \mathrm{~m})$. After 6 months of methotrexate treatment, a dramatic clinical improvement was observed. The haemodynamic response was less significant. The main limitation of our report is the absence of RHC evaluation immediately before methotrexate initiation, thus comparison after methotrexate treatment is more difficult and a delayed response to epoprostenol cannot be ruled out. Nevertheless, the following points suggest a role for immunosuppression: 1) the prolonged survival of this patient is exceptional regarding the unfavourable prognostic factors (severity of $\mathrm{PH}$ at baseline, 6MWD $<380 \mathrm{~m}$ and functional class $>$ II after 3 months of epoprostenol therapy) that predicted a survival of $<20 \%$ at 5 yrs [9]; 2) no increase in epoprostenol dose or addition of a new drug was required; and 3) the correlation of $\mathrm{PH}$ improvement with complete remission of LGL clonal expansion.

Moreover, in vitro and in vivo studies on serum from patients affected by PH and LGL leukaemia have provided robust data suggesting a strong aetiopathological link between these diseases. Two distinct (but not exclusive) mechanisms have been described. LAMY et al. [4] demonstrated that dysregulation of a Fas-mediated pro-apoptotic pathway resulted in Fas ligand (FasL) overexpression in serum from patients with LGL leukaemia and that this event could lead to Fas-/FasL-mediated apoptosis of pulmonary endothelial cells that constitutively express Fas on their surface. More recently, it was demonstrated that leukaemic T-cell LGLs from patients with PH constitutively exhibited direct and specific lytic activity against the pulmonary endothelial cell line CRL-2898 in vitro. Cytotoxicity was mediated by surface NKRs such as CD158b and CD94 overexpressed on leukaemic cells. Signalling events triggered by NKRs led to activation of transmembrane adaptor proteins DAP10 and DAP12 and subsequent activation of two intracellular signalling pathways: Ras/MEK/ERK and phosphatidyl inositol-3-kinase/ MEK/ERK, which led to survival and expansion of leukaemic cells due to Fas-mediated apoptosis resistance and to enhanced cytolytic activity against pulmonary endothelial cells, respectively $[6,7]$. Specific blockade of the Ras pathway with targeted therapy (the farnesyltransferase inhibitor tipifarnib) led to a decrease in endothelial cell toxicity in vitro and to an improvement in PH in a patient with associated natural killer-LGL [7].

In conclusion, our observations corroborate current data regarding a possible aetiopathological link between PH and T-cell LGL leukaemia. The efficacy of immunosuppressive treatment in this case highlights the importance of carefully identifying haematological disorders, such as T-cell LGL clonal expansion, when unexplained $\mathrm{PH}$ is diagnosed.

\section{O. Grossi*,\#, D. Horeau-Langlard", C. Agard*, A. Haloun", M. Lefebvre*, A. Neel* and M.A. Hamidou*}

*Service de Médecine Interne, Centre Hospitalier Universitaire, Hôtel-Dieu, \#Service de Médecine Interne et Infectiologie, Nouvelles Cliniques Nantaises, and 'Service de Pneumologie, Institut du Thorax, Centre Hospitalier Universitaire, Hôpital Nord Laënnec, Nantes, France.

Correspondence: O. Grossi, Service de Médecine Interne et Infectiologie, Nouvelles Cliniques Nantaises, 3 rue Eric Tabarly, 44277, Nantes, France. E-mail: o.grossi@free.fr

Statement of Interest: None declared.

\section{REFERENCES}

1 Simonneau G, Robbins IM, Beghetti M, et al. Updated clinical classification of pulmonary hypertension. J Am Coll Cardiol 2009; 54: Suppl. 1, S43-S54.

2 Harris NL, Jaffe ES, Stein H, et al. A revised European-American classification of lymphoid neoplasms: a proposal from the International Lymphoma Study Group. Blood 1994; 84: 1361-1392.

3 Lamy T, Loughran TP. How I treat LGL leukemia. Blood 2011; 117: 2764-2774.

4 Lamy T, Bauer FA, Liu JH, et al. Clinicopathological features of aggressive LGL leukaemia resemble Fas ligand transgenic mice. $\mathrm{Br} J$ Haematol 2000; 108: 17-23.

5 Chen X, Bai F, Sokol L. A critical role for DAP10 and DAP12 in CD8+ T cell-mediated tissue damage in large granular lymphocyte leukemia. Blood 2009; 113: 3226-3234.

6 Epling-Burnette PK, Sokol L, Chen X, et al. Clinical improvement by farnesyltransferase inhibition in NK large granular lymphocyte leukemia associated with imbalanced NK receptor signalling. Blood 2008; 112: 4694-4698.

7 Rossof LJ, Genovese J, Coleman M, et al. Primary pulmonary hypertension in a patient with $\mathrm{CD} 8 / \mathrm{T}$-cell large granulocyte leukemia. Amelioration by cladribine therapy. Chest 1997; 112: 551-553.

8 Howard LS, Chatterji S, Morrell NW, et al. Large granular lymphocyte leukaemia: a curable form of pulmonary arterial hypertension. Hosp Med 2005; 66: 364-365.

9 Sitbon $\mathrm{O}$, Humbert $\mathrm{M}$, Nunes $\mathrm{H}$, et al. Long term intravenous epoprostenol infusion in primary pulmonary hypertension: prognostic factors and survival. J Am Coll Cardiol 2002; 40: 7880-7888.

DOI: $10.1183 / 09031936.00014811$ 